\title{
Die Finanzierung der gesetzlichen Unfallversicherung: Chancen und Risiken bei Einführung von Kapitaldeckungselementen
}

\author{
Prof. Dr. Ulrich Becker, LL.M. (EHI)
}

\section{Einführung}

1. Zwei Prozesse haben dazu beigetragen, daß in den letzten Jahren die Rolle des Sozialstaats und damit die Ausgestaltung der sozialen Sicherungssysteme in Deutschland wie in den meisten anderen Mitgliedstaaten der Europäischen Union auf den Prüfstand gekommen ist, ${ }^{1}$ und daß insbesondere die gesetzliche Rentenversicherung bereits grundlegenden Reformen unterzogen wurde. ${ }^{2}$ Kurzfristig war es die schwierige Situation auf dem Arbeitsmarkt, die vor dem Hintergrund der zunehmenden Internationalisierung und des damit erhöhten Wettbewerbsdrucks ${ }^{3}$ das Bewußtsein für den Zusammenhang zwischen sozialer Sicherung und Arbeitskosten geschärft hat. ${ }^{4}$ Langfristig ist es die nicht mehr nur absehbare, ${ }^{5}$ sondern mittlerweile auch spürbar gewordene demographische Entwicklung, ${ }^{6}$ die insbesondere im Hinblick auf die künftige Finanzierung sozialer Sicherungssysteme zu Änderungsvorschlägen geführt hat.

Die Reformdebatten haben mittlerweile die gesetzliche Unfallversicherung (GUV) erreicht. ${ }^{7}$ Galt dieser Zweig der deutschen Sozialversicherung lange Zeit als zumindest verhältnismäßig krisenfest - wegen der Erfolge im Bemühen um die Senkung der Unfallzahlen, ${ }^{8}$ aber auch wegen der im Vergleich zur Kranken- und Rentenversicherung

1 Vgl. zur „europäischen Begleitung“ dieses Prozesses nur die Sozialpolitische Agenda, KOM (2005) 33 endg.; zur Debatte um die sog. „Nachhaltigkeit“ sozialer Sicherungssysteme Ebsen, Nachhaltigkeit soziale Sicherheit?, in: SDSRV 55 (2007), S. 79 ff.

2 Vgl. dazu nur Becker, Private und betriebliche Altersvorsorge zwischen Sicherheit und Selbstverantwortung, JZ 2004, S. $846 \mathrm{ff}$.

3 Dazu Becker/Schön (Hrsg.), Steuer- und Sozialstaat im europäischen Systemwettbewerb, 2005.

4 Ohne daß genaue und empirisch belegte Zahlen vorlägen, dazu Walwei/Zika, Arbeitsmarktwirkungen einer Senkung der Sozialabgaben, SF 2005, S. $77 \mathrm{ff}$.

$5 \mathrm{Zu}$ früheren Prognosen Kaufmann, Die Überalterung. Ursachen, Verlauf, wirtschaftliche und soziale Auswirkungen des demographischen Alterungsprozesses, 1960; Stolleis, Möglichkeiten der Fortentwicklung des Rechts der Sozialen Sicherheit zwischen Anpassungszwang und Bestandsschutz, DJT 1984, N, S. 9 ff.

6 Vgl. nur Becker, Die alternde Gesellschaft - Recht im Wandel, JZ 2004, S. 929 ff.

7 Während sich die Kommission für die Nachhaltigkeit in der Finanzierung der Sozialen Sicherungssysteme (auch bekannt als „Rürup-Kommission“) mit der GUV nicht eingehend beschäftigt hat, vgl. den Bericht unter http://www.soziale-sicherungssysteme.de/download/PDFs/Bericht.pdf. 
spürbar geringeren Ausgabenlast -, so hat sich das Bild vor allem in den letzten zwei Jahren verändert. 9

Denn mittlerweile geht es nicht mehr um die Frage, ob ein privates Versicherungssystem einem sozialen vorzuziehen sei. Diese Frage ist bekanntlich so alt wie die GUV selbst. ${ }^{10}$ Die zwischenzeitlichen Bestrebungen, eine Antwort zugunsten privater Versicherungsunternehmen mit Hilfe des europäischen Gemeinschaftsrechts zum Durchbruch $\mathrm{zu}$ verhelfen, ${ }^{11}$ hat sich durch die Rechtsprechung des EuGH ${ }^{12}$ weitgehend erledigt. ${ }^{13}$ Heute geht es statt dessen um weniger radikale, aber viel konkretere Aspekte. Im Mittelpunkt stehen dabei die Leistungen und die Organisation der Unfallversicherung. Aber selbst insofern bleibt immer die Frage im Hintergrund, wie die Finanzierung des Leistungssystems auf Dauer am besten gesichert werden kann - zumal, wie auf der Hand liegt, Leistungen und Organisation ihrerseits natürlich die finanzielle Basis wesentlich beeinflussen.

2. Im folgenden möchte ich zunächst die aktuellen Entwicklungen aufzeigen (II.). Dabei sollen sowohl die unterschiedlichen Positionen, die in der Reformdebatte von interessierten Verbänden zum Pro und Contra einer völligen oder teilweisen Umstellung auf eine Kapitaldeckung vorgetragen werden, als auch der Gesetzentwurf, der voraussichtlich demnächst zu einer Reform führen wird, vorgestellt werden. Im Anschluß geht es um die Grundlagen, nämlich das gegenwärtige Finanzierungssystem der gesetzlichen Unfallversicherung in Deutschland, das in seinen Strukturen vorgestellt werden soll (III.). In einem dritten Schritt werden sich die Ausführungen dann den Vor- und Nachteilen der Einführung von Kapitaldeckungselementen zuwenden (IV.). Dabei sollen aber nicht ökonomische Argumente im Vordergrund stehen, sondern vor allem die normative Frage, ob und in welcher Weise eine Einbindung solcher Elemente mit dem bestehenden System vereinbar ist.

8 Zum Rückgang der Arbeitsunfälle etwa BMA, Die gesetzliche Unfallversicherung in der Bundesrepublik Deutschland im Jahre 2000, 2002, T21. Das hat sich zwar jüngst insofern geändert, als 2006 mehr Arbeitsunfälle als 2005 zu verzeichnen waren, was aber auf die steigende Zahl der Beschäftigten zurückzuführen ist, vgl. http://www.dguv.de/inhalt/zahlen/au_wu/index.jsp.

9 Dazu, daß früher die GUV regelmäßig nicht im Mittelpunkt des öffentlichen Interesses stand, $v$. Maydell, Die gesetzliche Unfallversicherung - ein übertragbares Modell?, in: FS für Waltermann, 1996, S. 99.

10 Vgl. zur Entstehung und zu den damaligen Diskussionen, ob der Versicherungszwang bzw. der Verzicht auf Gewinne eine private Trägerschaft ausschlossen, Vogel, Bismarcks Arbeiterversicherung, 1951, S. $152 \mathrm{ff}$.

11 Grundl. zu den dahinter stehenden gemeinschaftsrechtlichen Fragen Giesen, Sozialversicherungsmonopol und EGV, 1995.

12 EuGH v. 22.1.2002, Rs. C-218/00 (INAIL), Slg.2002, S. I-691.

13 Vgl. aber auch Giesen, Das BSG, der EG-Vertrag und das deutsche Unfallversicherungsmonopol, ZESAR 2004, S. 151 ff.; Seewald, Kein Monopol der gesetzlichen Unfallversicherung, SGb 2004, S. 387 ff. und 453 ff.; gegen beide Fuchs, Die Konformität des Unfallversicherungsmonopols mit dem Gemeinschaftsrecht, SGb 2005, S. 65 ff. 


\section{Aktuelle Entwicklungen}

\section{Positionen für und gegen eine Reform}

„Ist nach alledem die versicherungstechnische Zweckmäßigkeit des Umlageverfahrens unter den sozialpolitischen und wirtschaftlichen Aspekten der heutigen industriellen Gesellschaft nahezu unbestritten ..."14 - diese Anfang der 1960er Jahre getroffene Feststellung ist mittlerweile eher in ihr Gegenteil verkehrt worden.

Die Stimmen, die in der letzten Zeit eine völlige oder teilweise Umstellung des gegenwärtigen Finanzierungssystems auf eine Kapitaldeckung befürworten, mehren sich. Die Gründe dafür sind durchaus unterschiedlich. Am weitestgehenden ist das Argument, auf Grund der Alterung und gleichzeitigen Schrumpfung der Bevölkerung sei eine Umstellung erforderlich. In diesem Sinne fordern z.B. die Bundesvereinigung der Deutschen Arbeitgeberverbände (BDA) ${ }^{15}$ und der Deutsche Industrie- und Handelskammertag (DIHK) ${ }^{16}$ die Einführung der Kapitaldeckung: Nur so ließe sich eine übermäßige Belastung zukünftiger Generationen vermeiden. Der DIHK geht einen Schritt weiter und tritt gleich für die Aufgabenübertragung an eine private Unfallversicherung ein, da bei der Rentenversicherung schlechte Erfahrungen mit einem Kapitaldeckungsverfahren im staatlichen Sozialversicherungssystem gemacht worden seien. Die „Privatisierung der Wegeunfälle“" sei in dieser Hinsicht ein erster Schritt hin zu einem kapitalgedeckten, privaten Unfallschutz. ${ }^{17}$

Konkreter an der wirtschaftlichen Situation der vergangenen Jahre und an dem gesunkenen Beitragsaufkommen knüpfen andere Stimmen an. So meinen etwa der Hauptverband des Deutschen Einzelhandels (HDE) ${ }^{18}$ und die FDP ${ }^{19}$, ein Kapitaldeckungsverfahren sei deswegen unumgänglich, weil die Finanzierung der Altrenten von einem Umlagesystem nicht angemessen zu bewältigen sei.

Im Hinblick auf den letztgenannten Punkt, die finanziellen Aufwendungen für Renten, befürwortet auch der Verband der Träger der gesetzlichen Unfallversicherung, die

14 Rengert, Finanzierungsprobleme der Unfallversicherung, in: FS für Lauterbach, 1961, S. 95, 101.

15 Positionspapier Generalüberholung überfällig! Leistungen konzentrieren - Effizienz steigern, Forderungen zur Reform der gesetzlichen Unfallversicherung, http://bda-onlne.de/www/bdaonline.nsf/id/Reformvorschlaegezurgesetzlich1/\$file/PopaUnfallversicherung6_06.pdf, S. 3, 8.

16 Transparenz, Verlässlichkeit und Effizienz - Agenda für eine zukunftssichere Ausgestaltung der gesetzlichen Unfallversicherung in Deutschland, http://www.dihk.de/inhalt/download/unfallversicherung.pdf, S. 3, 4 ff., 11.

17 A.a.O., S. 3, 5, 11.

18 Reform der gesetzlichen Unfallversicherung, http://www.einzelhandel.de/servlet/PB/menu/1058331/index.html, S. 8 ff., 16.

19 Positionspapier „Mehr Wettbewerb und Kapitaldeckung in der Unfallversicherung“, http://www.fdp-fraktion.de/files/723/Positionspapier_Unfallversicherung.pdf, S. $1 \mathrm{f}$. 
Deutsche Gesetzliche Unfallversicherung (DGU) ${ }^{20}$, grundsätzlich einen Übergang zu einem Finanzierungssystem mit Kapitaldeckungselementen, die Einführung eines Rentenwert-Umlageverfahrens.

Ganz anderer Ansicht ist die Bund-Länder-Arbeitsgruppe, die sich mit der Reform der Unfallversicherung beschäftigt hat. Sie kam zu dem Ergebnis, daß die Altlasten nur durch „eine solidarische Kraftanstrengung der Unternehmer“ in einem Umlagesystem bewältigt werden können. Zudem führe auch nur das Umlageverfahren zu „einem gerechten und solidarischen Ausgleich zwischen den verschiedenen Branchen und Risikogruppen", wovon nicht zuletzt die Unternehmen durch eine geringere finanzielle Einzelbelastung profitierten. ${ }^{21}$ Ein Nachteil der Umstellung auf ein Kapitaldeckungsverfahren wären außerdem deutlich höhere Beiträge in der Gegenwart. Auf erhebliche zusätzliche Belastungen für Mitgliedsunternehmen der Berufsgenossenschaften wies auch der Hauptverband der gewerblichen Berufsgenossenschaften (HVBG) ${ }^{22}$ hin.

Zusätzlich zu möglichen Beitragserhöhungen wird in einem im Auftrag des Bundesministeriums für Arbeit und Soziales (BMAS) erstellten Gutachten zur Reform der GUV 23 davor gewarnt, daß im Falle einer Systemumstellung Beitragsanteile in der Gegenwart nicht zur Verfügung stünden. Zudem würden erhebliches Kapital gebunden sowie enorme Rückstellungen entwickelt und damit der Wirtschaft viel Kapital entzogen. ${ }^{24}$

Diesen Bedenken halten die Arbeitsgemeinschaft Selbständiger Unternehmer $(\mathrm{ASU})^{25}$, der Zentralverband des Deutschen Handwerks ${ }^{26}$ sowie die Arbeitsgemeinschaft der Fachverbände des baden-württembergischen Handwerks ${ }^{27}$ entgegen, daß schon eine schrittweise Teilumstellung genügen würde und daß der notwendige Kapitalstock in kleinen Schritten und bei gleichzeitigem Ausschöpfen vorhandener Einspar-

20 Positionspapier „Reform des Rechts der gesetzlichen Unfallversicherung“, http://www.bgdp.de/pages/presse/pospapier_guv.pdf, S. 8.

21 Eckpunkte zur Reform der gesetzlichen Unfallversicherung Beschluss vom 29.6.2006, http://www.rguvv.de/fileadmin/download/info_plus/EckpunkteEndfassung.pdf, S. 2 f.

22 Reformen gestalten - überzeugen durch Handeln, www.hvbg.de/d/pages/service/download/jahrbuch/jb/pdf/jb2003.pdf, S. 12.

23 Gutachten zur Neuorganisation der gesetzlichen Unfallversicherung, Gutachten von Rürup und Steinmeyer v. 30. März 2006, http://www.bgchemie.de/files/37/Gutachten_GUV_2006-03-30.pdf, S. 48.

24 Die Argumente sind bekannt; dazu und zu möglichen Lösungen näher Heubeck $A G$, Versicherungsmathematisches Gutachten zur Finanzierung der gewerblichen Unfallversicherung v. 24.4.2002, S. $51 \mathrm{ff}$.

25 Positionspapier „Reform der gesetzlichen Unfallversicherung“, http://www.asu.de/www/doc/fff38493f5a1643ee8ef247750540ee2.pdf, S. 7.

26 Positionspapier „Reform der gesetzlichen Unfallversicherung“, http://www.zdh.de/sozial-undtarifpolitik/unfallversicherung-arbeitsschutz/reform-der-unfallversicherung-aktualisiertespositionspapier-vom-januar-2007.html, S. $3 \mathrm{f}$.

27 Positionspapier zur Reform der gesetzlichen Unfallversicherung, http://www.handwerk-bw.de/fileadmin/gruppe_arbeitundsoziales/Datei_Upload/ArgeFVBWHT_ReformBG_kurz.pdf, S. 1, 5. 
potentiale aufgebaut werden sollte, um die derzeitigen Beitragszahler nicht zu überlasten. Auf längere Sicht könne so eine zukunftssichere Finanzierung entwickelt werden, und die Kosten für die Finanzierung der Unfallrenten würden sich durch die Verzinsung des Kapitalstocks verringern.

Im Übrigen bringt der DIHK gegenüber den Bedenken, die mit der Einführung eines Kapitaldeckungsverfahrens verbundenen Belastungen seien zu hoch, in dem bereits genannten Positionspapier ${ }^{28}$ vor, daß die Ausgaben durch eine Verkleinerung des Leistungskatalogs begrenzt werden könnten. Gegen solche Leistungskürzungen wehren sich jedoch nicht zuletzt die Gewerkschaften nach Kräften.

Damit schließt sich der Kreis. Der Zusammenhang zwischen der Art der Finanzierung und ihrem Gegenstand, zwischen Finanzierungsverfahren und Leistungen, rückt in den Blickpunkt - und genau an diesem Punkt ist die Reform im Juli 2007 vorerst gescheitert.

\section{Zum Entwurf eines Unfallversicherungsmodernisierungsgesetzes (UVMG)}

Im April 2007 hatte das BMAS den vierten Arbeitsentwurf zur Reform der gesetzlichen Unfallversicherung vorgelegt. Der Arbeitsentwurf, der von einer Bund-LänderArbeitsgruppe erarbeitet wurde, besteht aus zwei Teilen, die den unterschiedlichen Reformbereichen entsprechen: So behandelt der erste Teil die Organisationsreform der gesetzlichen Unfallversicherung, der zweite Teil ${ }^{29}$ hingegen baut auf dem EckpunktePapier der Bund-Länder-Arbeitsgruppe vom 29. Juni $2006^{30}$ auf und befaßt sich mit der Leistungsreform der gesetzlichen Unfallversicherung. Auf der Grundlage der Arbeitsentwürfe ist dann ein Referentenentwurf eines UVMG ${ }^{31}$ ausgearbeitet und Ende November 2007 publiziert worden. Im Februar 2008 wurde der Entwurf vom Kabinett beschlossen 32 und dem Bundesrat zugeleitet. ${ }^{33}$

Hervorzuheben ist zunächst, daß sich der Referentenentwurf wieder auf eine Organisationsreform beschränkt. Die Reform des Leistungsrechts - die im Juni 2007 an massiver Kritik von Seiten der Gewerkschaften gescheitert ist - wurde (zumindest vorerst) zurückgestellt. ${ }^{34}$ Insbesondere die Fragen der künftigen Regelung der Rentenvorausset-

28 Vgl. Fußn. 16.

29 http://www.vbw-bayern.de/agv/Unsere_Themen-Sozialpolitik-Arbeit-Mehr_zum_ThemaArbeitsentwurf_zur_Reform_der_gesetzlichen_Unfallv-7801.htm?PHPSESSID=eaec0b7485c4a7bef7 $\overline{140633299 f c 53 e . ~}$

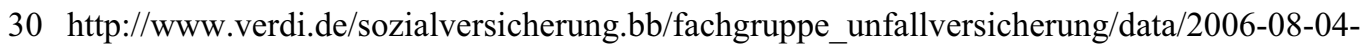
Synopse\%20Eckpunkte-nach\%20vorstand.pdf.

$31 \mathrm{http} / / / \mathrm{www}$.gutearbeit-online.de/archiv/hintergrund/2007/referentenentwurf_uv.pdf.

32 Dazu und zur Fassung des Entwurfs http://www.bmas.de/coremedia/generator/24850/uvmg_entwurf.html.

33 BR-Drs. 113/08.

34 Krit. dazu Beschluß des Bundesrats v. 25.4.2008, BR Drs. 113/08 (B). 
zungen und der Ausgliederung von Wegeunfällen bleiben damit offen. Kernpunkt der Organisationsreform soll nach dem Referentenentwurf die Reduzierung der Unfallversicherungsträger auf höchstens neun Berufsgenossenschaften und 17 Unfallkassen sein, um „nachhaltig leistungsfähige Träger“ zu schaffen. ${ }^{35}$ Die nötigen Fusionen sollen von der Selbstverwaltung in Eigenregie konzipiert und durchgeführt werden. Parallel dazu sollen viele Aufgaben in dem Spitzenverband, der Deutschen Gesetzlichen Unfallversicherung, zentralisiert werden. Zweck dieser Maßnahmen sind die Modernisierung der Verwaltung sowie eine Eindämmung der Beitragsspreizungen zwischen den einzelnen gewerblichen Berufsgenossenschaften. ${ }^{36}$ Verzichtet wurde darauf, einen Spitzenverband in der Rechtsform einer Körperschaft des öffentlichen Rechts zu schaffen. Die Unfallversicherungsträger hatten sich dagegen gewehrt und auch verfassungsrechtliche Bedenken vorgetragen. ${ }^{37}$ Nachdem sich der Hauptverband der gewerblichen Berufsgenossenschaften (HVBG) und der Bundesverband der Unfallversicherungsträger der öffentlichen Hand (BUK) zur Deutschen Gesetzlichen Unfallversicherung (DGUV) zusammengeschlossen hatte, ${ }^{38}$ wird es bei der von der Selbstverwaltung gewählten Form des privatrechtlichen Vereins bleiben, wenn auch im Hinblick auf dessen Aufgabenstellungen - wie in den meisten anderen Sicherungszweigen auch - eine Zentralisierung unübersehbar ist, die mit einer Fachaufsicht des Ministeriums einhergeht. 39

Ein zweiter Schwerpunkt des Referentenentwurfs liegt auf Änderungen im Vermögensrecht und der Neuverteilung der Rentenaltlasten. Das neue Verfahren soll „die solidarische Lastenverteilung zwischen den Gewerbezweigen nachhaltig“" stärken. ${ }^{40}$ Es unterscheidet sich von dem bisherigen Lastenausgleichsverfahren ${ }^{41}$ durch die Einführung eines sog. Überaltlastausgleichs, wie er zwischenzeitlich von den Trägern beschlossen worden ist. ${ }^{42}$ Künftig trägt jede Berufsgenossenschaft ,jährlich Rentenlasten in Höhe des 5,5fachen ihrer Neurenten für Arbeitsunfälle und des 3,4fachen ihrer mit dem Latenzfaktor gewichteten Neurenten für Berufskrankheiten"..43 Die darüber hinausgehenden Rentenlasten tragen alle Träger gemeinsam. ${ }^{44}$

35 Entwurf (Fußn. 32), S. 59.

36 Entwurf (Fußn. 32), S. 59 f.

37 Dazu näher Ruland, Verein oder Körperschaft?, 2007.

38 Durch Beschlüsse der Mitgliederversammlungen mit Wirkung ab dem 1.6.2007.

39 Vgl. den geplanten $\S 87$ Abs. 3 SGB IV, Entwurf (Fußn. 32), S. 39.

40 Entwurf (Fußn. 32), S. 61.

41 Dazu unten, III. 1.

42 Vgl. Beschluss der Mitgliederversammlung 2/2006 des HVBG v. 1.12.2006 zur Reform der gesetzlichen Unfallversicherung - Konzept zur Lastenverteilung; vgl. auch die Stellungnahme der BDA, http://www.bda-online.de/www/bdaonline.nsf/id/817F1CC601F6973FC12573EF0060E695/\$file/StNKabEUVMG.pdf, S. 7 ff.; vgl. bereits das Heubeck-Gutachten (Fußn. 24), S. 6 f.

$43 \S 178$ Abs. 1 S. 1 SGB VII des Entwurfs; zur Anpassung der Werte $\S 178$ Abs. 1 S. 2.

$44 \S 178$ Abs. 2 SGB VII des Entwurfs, und zwar in einer vom Eckpunktepapier abweichenden Gewichtung, nämlich zu $70 \%$ nach dem Verhältnis der Arbeitsentgelte und zu $30 \%$ nach dem Verhältnis der Neurenten. 
Zudem werden Änderungen für die Bildung von Betriebsmitteln und Rücklagen angestrebt: Die Betriebsmittel ${ }^{45}$ sind künftig ebenso wie die Rücklagen ${ }^{46}$ beschränkt. Darüber hinaus sollen die Träger verpflichtet werden, „Altersrückstellungen für die bei ihnen beschäftigten Arbeitnehmerinnen und Arbeitnehmer, denen eine Anwartschaft auf Versorgung nach beamtenrechtlichen Vorschriften oder Grundsätzen gewährleistet wird, zu bilden“ , 47 ebenso für die Erfüllung von unmittelbaren Zusagen auf Leistungen der betrieblichen Altersvorsorge gegenüber ihren Tarifbeschäftigten. ${ }^{48}$ Durch Rechtsverordnung soll „,das Nähere zur Höhe der für die Altersrückstellungen erforderlichen Zuweisungssätze, zum Zahlverfahren der Zuweisungen sowie zur Überprüfung der Höhe der Zuweisungssätze“" geregelt werden. ${ }^{49}$ Soweit die Zuständigkeit für den Erlaß einer entsprechenden Verordnung auf das Bundesversicherungsamt übertragen wird, ist eine Mitwirkung der Selbstverwaltung in Form einer Anhörung der Spitzenverbände vorgesehen. ${ }^{50}$ Darüber hinaus werden die Träger der Unfallversicherung aber großen Einfluß nicht nur auf die Neuorganisation der Unfallversicherung, sondern insbesondere auch auf die Modalitäten für die Bildung der Altersrückstellungen nehmen können. Denn der Gesetzentwurf sieht vor, daß die DGUV gemeinsam mit dem Spitzenverband der landwirtschaftlichen Sozialversicherung ein Konzept zur Einführung dieser Rückstellungen bis zum 30. April 2009 vorlegt. 51

\section{Grundlagen: Das Finanzierungssystem}

\section{Grundsätze}

Die gesetzliche Unfallversicherung wird ausschließlich durch Beiträge der Unternehmer finanziert. Das entspricht - soweit damit die Beitragsfreiheit der Arbeitnehmer

$45 \S 172$ Abs. 1 SGB VII des Entwurfs: „Betriebsmittel dürfen nur verwendet werden 1. für Aufgaben, die gesetzlich oder durch die Satzung vorgesehen sind, sowie für die Verwaltungskosten, 2. zur Auffüllung der Rücklage und zur Bildung von Verwaltungsvermögen.“

$46 \S 172$ a Abs. 2 SGB VII des Entwurfs: „Die Rücklage wird mindestens in zweifacher Höhe der durchschnittlichen monatlichen Ausgaben des abgelaufenen Kalenderjahres und höchstens bis zur vierfachen Höhe der durchschnittlichen monatlichen Ausgaben des abgelaufenen Kalenderjahres gebildet; Stichtag für die Bemessung ist der 31. Dezember des laufenden Kalenderjahres.“

$47 \S 172 \mathrm{c}$ Abs. $1 \mathrm{~S} .1 \mathrm{SGB}$ VII des Entwurfs.

$48 \S 172 \mathrm{c}$ Abs. 1 S. 2 SGB VII des Entwurfs.

$49 \S 172$ c Abs. 3 S. 1 SGB VII des Entwurfs. Zuständig für deren Erlaß ist das BMAS, das die Befugnis auf das Bundesversicherungsamt übertragen kann.

$50 \S 172$ c Abs. 3 S. 3, 1. HS SGB VII des Entwurfs: „Rechtsverordnungen, die nach Satz 2 erlassen werden, bedürfen einer Anhörung der Deutschen Gesetzlichen Unfallversicherung e.V. sowie des Spitzenverbandes der landwirtschaftlichen Sozialversicherung ...".

$51 \S 219 \mathrm{a}$ Abs. 2 SGB VII des Entwurfs. 
angesprochen ist - der Funktion dieses Versicherungszweigs: Die Risikoabsicherung gegen Arbeitsunfälle und Berufskrankheiten dient der Ablösung der Arbeitgeberhaftung. Die gesetzliche Unfallversicherung löst die Haftung aus dem privatrechtlichen Austauschverhältnis und überführt sie in das öffentliche Verwaltungsrecht. ${ }^{52}$ Das soll Sicherheit des Schutzes durch Insolvenzfestigkeit garantieren und den Betriebsfrieden durch Bürokratisierung bewahren. Dieser Ansatz wird in Deutschland insofern konsequent betrieben, als Kehrseite des Schutzes der weitgehende Ausschluß der privatrechtlichen Haftung von Arbeitgebern und Arbeitskollegen ist. ${ }^{53}$

Bei dieser Anlage ist die Abgrenzung der Zuständigkeit der Unfallversicherung zugleich die Abgrenzung der Finanzierungsverantwortung der Arbeitgeber. Diese Abgrenzung wird rechtsdogmatisch über den sog. rechtlichen inneren Zusammenhang vorgenommen. $\mathrm{Ob}$ ein solcher Zusammenhang vorliegt oder nicht entscheidet über die $\mathrm{Zu}-$ rechnung eines unfall- oder krankheitsverursachenden Ereignisses zu einer betrieblichen oder einer eigenwirtschaftlichen Tätigkeit. ${ }^{54}$ Kann die Zurechnung erfolgen, hat die Unfallversicherung für die Folgen von Unfall und Krankheit einzustehen. Fehlt es an dem rechtlichen Zusammenhang, hat die Krankenversicherung die notwendigen Sozialleistungen zu erbringen.

Bis heute ist es bei der damit skizzierten Finanzierungsverantwortung der Arbeitgeber geblieben. Ein Bundeszuschuß wird nicht gezahlt. ${ }^{55}$ Auch das ist ein Grundsatz, welcher der ursprünglichen Konzeption des Unfallversicherungssystems entspricht wenn er auch bei der Einführung des Versicherungszweigs höchst umstritten war ${ }^{56}$ und zeitweise Durchbrechungen erfahren hat.

Das angewendete Finanzierungsverfahren ist ein Umlageverfahren. ${ }^{57}$ Es wird in Form der sog. nachträglichen Bedarfsdeckung durchgeführt, ${ }^{58}$ d.h. der durch die Bei-

52 Rengert, in: FS für Lauterbach (Fußn. 14), S. 96; vgl. auch Wertenbruch, Sozialverfassung - Sozialverwaltung, 1974, S. 135.

53 Nach Maßgabe der $\S \S 104$ ff. SGB VII. Vgl. dazu auch BVerfGE 34, 118; BVerfG [Kammer], NJW 1995, S. $1607=$ SozR 3-2200 § 636 Nr. 1.

54 Vgl. zur st. Rspr. des BSG nur Krasney, in: Brackmann (Hrsg.), Handbuch der Sozialversicherung, GUV, Stand 2002, § 8, Rdnr. $23 \mathrm{ff}$.

55 Auf die Frage der Auferlegung von Sonderlasten durch die Bewältigung der Kriegsfolgen und der Übernahme der Lasten aus der ehemaligen DDR soll hier nicht näher eingegangen werden, denn dabei geht es um die Abgrenzung der Verantwortlichkeit der Allgemeinheit von jener der Unfallversicherung; vgl. zu den DDR-Altlasten BSGE 79, 23 = SozR 3-8110 Kap. VIII J III Nr. 1, Nr. 1; BSG, SozR 3-8110 Kap. VIII J III Nr. 1, Nr. 2.

56 Insbesondere Bismarck hatte ursprünglich einen anderen Ansatz verfolgt, vgl. nur Stolleis, Historische Grundlagen - Sozialpolitik bis 1945, in: Geschichte der Sozialpolitik, 2001, Bd. 1, S. 199, $246,250 \mathrm{f}$.

57 Und zwar hier bezogen auf die sog. Eigenumlage, d.h. ohne Berücksichtigung des Lastenausgleichs, vgl. Ricke, in: KassKomm., § 152 SGB VII, Rdnr. 3.

58 Vgl. § 152 Abs. 1 SGB VII; zu dieser Eigenheit der UV Platz, Aufbringung der Mittel, in: Schulin (Hrsg.), HS-UV, 1996, § 58, Rdnr. 6. 
träge zu deckende Finanzbedarf, das Umlagesoll (US), 59 wird retrospektiv bestimmt. Dieser Bedarf besteht aus dem im abgelaufenen Kalenderjahr festgestellten Gesamtbedarf (GB), ${ }^{60}$ vermindert um die Verwaltungseinnahmen (VE).

Bei der Umlage auf die Unternehmen, also der Verteilung der Finanzierungslast zwischen den Arbeitgebern, ist von besonderer Bedeutung, daß der Beitrag, der von dem einzelnen Unternehmer zu entrichten ist $\left(\mathrm{B}_{\mathrm{U}}\right)$, nicht nur nach den Arbeitsentgelten (AE) im Unternehmen 61 - oder ausnahmsweise den im Unternehmen beschäftigten Personen (sog. Kopfbeitrag $)^{62}$ - berechnet wird. Er wird vielmehr auch je nach Unfallgefahr abgestuft. Zu diesem Zweck werden verschiedene Gefahrklassen (GK) gebildet.

Aus der Multiplikation der Arbeitsentgelte (bzw. Kopfsumme) mit der oder den jeweils anwendbaren Gefahrklasse(n) ergibt sich die Beitragseinheit je Unternehmen $\left(\mathrm{BE}_{\mathrm{U}}\right)$. Diese Beitragseinheit ist mit dem sog. Beitragsfuß (BF) zu multiplizieren, der wiederum dadurch gewonnen wird, daß das Umlagesoll durch die Beitragseinheiten aller Unternehmen $\left(\mathrm{BE}_{\mathrm{G}}\right)$ dividiert wird. ${ }^{63}$ Formelhaft kann dies folgendermaßen ausgedrückt werden:

$$
\mathrm{B}_{\mathrm{U}}=\left[\mathrm{BF}=\frac{(\mathrm{US}=\mathrm{GB}-\mathrm{VE})}{\left(\mathrm{BE}_{\mathrm{G}}=\mathrm{AE}_{\mathrm{G}} \times \mathrm{GK}\right)}\right] \times \quad\left[\mathrm{BE}_{\mathrm{U}}=\mathrm{AE}_{\mathrm{U}} \times \mathrm{GK}\right]
$$

\section{Risikobezug}

Damit ist bereits gesagt, daß sich die Beiträge zur gesetzlichen Unfallversicherung auch nach dem in einem Unternehmen herrschenden Risiko richten. Zu diesem Zweck setzen die Berufsgenossenschaften durch Satzung Gefahrtarife fest. ${ }^{64}$ Dabei sind Tarifstellen zu bilden (§ 157 Abs. 2 S. 1 SGB VII), in denen Risiko- oder Gefahrengemeinschaften zusammengefaßt werden. In der Praxis erfolgt diese Gliederung im Wesentlichen nach Gewerbezweigen bzw. Gewerbezweiggruppen, ${ }^{65}$ ergänzt durch eine Diffe-

$59 \S 153$ Abs. 1 SGB VII.

60 Vgl. § 152 Abs. 1 S. 2 SGB VII. Gemeint sind die Kosten, die den Berufsgenossenschaften durch die Erfüllung ihrer gesetzlichen Aufgaben entstehen, die Aufwendungen für die Verwaltung sowie den sog. Finanzdienst (Betriebsmittel, § 171 SGB VII) und die Mittel zur Ansammlung von Rücklagen (§ 172 SGB VII).

61 Bis zur Höhe des Höchstjahresarbeitsverdienstes, § 153 Abs. 2 SGB VII. Zur Berechnung nach dem Jahresarbeitsverdienst (= Versicherungssumme) für bestimmte Personen § 154 SGB VII.

62 Sofern dies die Satzung bestimmt, vgl. § 155 SGB VII; zur Schätzung beim Kopfbeitrag BSG v. 18.4.2000, B 2 U 2/99 R.

63 Vgl. $\S 153$ Abs. 1, 167 SGB VII.

$64 \S 157$ I 1 SGB VII; zur Genehmigungspflicht § 158 SGB VII.

65 Vgl. Schulz, Verfassungsrechtliche Fragen der Festsetzung der Beiträge in der gesetzlichen Unfallversicherung, SGb 1999, S. 172, 173. 
renzierung nach Tätigkeiten. 66 Zusammengeschlossen werden müssen grundsätzlich strukturell und technologisch gleichartige Betriebe. ${ }^{67}$ Weisen Gewerbezweige nicht eine geforderte Mindestgröße auf, kommt eine Zusammenfassung von Betrieben neben dem Technologieprinzip auch nach dem Belastungsprinzip in Betracht, d.h. Gewerbezweige mit statistisch vergleichbarer Belastung können eine eigene Tarifstelle bilden. ${ }^{6}$

Die Tarifstellen bestimmen also Untergruppen von Unternehmen und damit innerhalb der Zuständigkeit einzelner Berufsgenossenschaften einzelne Solidargemeinschaften. Auf diese wird dann die Beitragslast entsprechend dem Äquivalenzprinzip verteilt. Auch dazu ein Blick auf die angewandte Technik: Den Tarifstellen werden Gefahrklassen zugeordnet, und zwar nach dem Verhältnis der gezahlten Leistungen zu den Arbeitsentgelten ( $\$ 157$ III SGB VII). Relevant sind dafür die im Beobachtungszeitraum tatsächlich entstandenen Entschädigungslasten. ${ }^{69}$ Die Unternehmen werden zu den Gefahrklassen veranlagt ( $\$ 159$ SGB VII). Ein Unternehmen kann einer Gefahrklasse zugeordnet sein, bei verschiedenen Tätigkeiten (im Falle des sog. gemischten Unternehmens) mehreren Gefahrklassen. Nur in ganz engem Rahmen sind abweichend von der jeweils relevanten Gefahrklasse Erhöhungen oder Herabsetzungen zulässig. ${ }^{70}$ Dennoch wirkt sich auch das konkrete Unfallgeschehen in einem Unternehmen auf die zu zahlenden Beiträge aus: Die Berufsgenossenschaften müssen unter Berücksichtigung der anzuzeigenden Versicherungsfälle Zuschläge auferlegen oder Nachlässe bewilligen (§ 162 SGB VII). Dieses Beitragsausgleichsverfahren „belohnt“ ein erfolgreiches Bemühen um Verhinderung von Versicherungsfällen und „bestraft" umgekehrt unfall- und krankheitsträchtiges Verhalten. Es verfolgt damit das Ziel, die Prävention durch monetäre Anreize zu verbessern. ${ }^{71}$

Betrachtet man die vorstehend zusammengefaßten Grundsätze, läßt sich festhalten, daß in der Unfallversicherung sehr viel stärker als in den anderen Zweigen der deutschen Sozialversicherung eine Äquivalenzbeziehung zwischen individuellem Risiko und dem Sozialversicherungsbeitrag besteht, was zu der Feststellung führt, in der Un-

66 Vgl. dazu Ricke, in: KassKomm., § 157 SGB VII, Rdnr. 9 f. Vgl. zur Mischung auch BSG, NZA, 1992, S. 335.

67 Platz, Aufbringung der Mittel, in: HS-UV (Fußn. 58), § 58, Rdnr. 38 ff. Vgl. zur Kritik wegen des z.T. nicht für ausreichend gehaltenen Risikobezugs Gitter, Die gesetzliche Unfallversicherung nach der Einordnung ins Sozialgesetzbuch - ein Versicherungszweig ohne Reformbedarf?, BB 1998, Beil. 6, S. 14 m.w.N.

68 Ricke, in: KassKomm., § 157 SGB VII, Rdnr. $11 \mathrm{f}$.

69 Vgl. näher dazu und zum Erfordernis der Berechnung Ricke, a.a.O., Rdnr. 14.

70 Bezogen auf erheblich abweichende Risiken, vgl. Ricke, a.a.O., Rdnr. 17.

71 Richtig ist, dass die Unfallgefahr vom Unternehmer nicht vollumfänglich beeinflusst werden kann; daraus wird z.T. geschlossen, durch die Bildung von Gefahrklassen würden Unternehmer ungleich behandelt, und diese Ungleichbehandlung müsse durch das Verhältnismäßigkeitsprinzip begrenzt werden, so Fenn, Effektiver Rechtsschutz gegen Gefahrtarife, SGb 2004, S. 94, 95. Diese Folgerung setzt aber voraus, dass das Äquivalenzprinzip durch das Solidarprinzip von vornherein begrenzt wird, eine Annahme, die aus den Strukturen des Unfallversicherungssystems ihrerseits erst abgeleitet werden muss. 
fallversicherung sei das Versicherungsprinzip relativ stark ausgeprägt. ${ }^{72}$ Das hat vor dem Hintergrund der Annahme, die Differenzierung zwischen Privat- und Sozialversicherung nach Funktion und Ausgestaltung sei nicht nur eine ordnungspolitisch zu verfolgende Aufgabe, sondern auch eine verfassungs- und gemeinschaftsrechtliche Notwendigkeit, Anlaß zu Kritik gegeben. Zumindest bei zu weitgehender Orientierung der Beiträge an den Gefahrklassen komme der soziale Ausgleich zu kurz, der aber jede Sozialversicherung prägen müsse. Es bedürfe also eines „gewissen Solidarausgleichs“, um die Unfallversicherung überhaupt (sowohl kompetenzmäßig als auch im Hinblick auf die mit ihr verbundene Zwangsmitgliedschaft ${ }^{73}$ ) zu rechtfertigen. ${ }^{74}$ Daraus wird dann geschlossen, Gefahrtarife könnten nur mit Einschränkungen für verfassungskonform gehalten. ${ }^{75}$ In ähnliche Richtung, wenn auch mit potentiell weiterreichenden Konsequenzen, geht der gemeinschaftsrechtliche Einwand: Ließen sich die Aufgaben der Unfallversicherungsträger durch privatrechtliche Unternehmen ebenso gut durchführen, seien die mit einem Versicherungsmonopol verbundenen Beschränkungen der im EGV garantierten Dienstleistungsfreiheit 76 nicht zu rechtfertigen. ${ }^{77}$

Für den gemeinschaftsrechtlichen Einwand genügt es, auf die jüngere Rechtsprechung des EuGH zu verweisen. ${ }^{78}$ Aber auch der verfassungsrechtliche Einwand, der sich zunächst nur gegen eine weitgehende Risikoorientierung wendet, ist - selbst bei prinzipiellem Festhalten an dem einer Sozialversicherung eigenen Wesensmerkmal des sozialen Ausgleichs ${ }^{79}$ - nicht überzeugend, ${ }^{80}$ sofern die bestehenden gesetzlichen Vorschriften über die Beitragsberechnung und ihre praktische Anwendung in den Blick genommen werden. Denn der Risikobezug kann innerhalb der Berufsgenossenschaften durch Höchst- und Niedrigstgefahrklassen für die Gefahrtarife beschränkt werden; vielfach werden die Gefahrklassen nach den Neulasten berechnet, ${ }^{81}$ womit sich zumindest ein Risikoausgleich zwischen den Tarifstellen im Hinblick auf die Altlasten ergibt. ${ }^{82}$ Gerade unter Berücksichtigung der Spannweite der unterschiedlichen Durchschnittsbei-

72 Vgl. F. Kirchhof, Das Solidarprinzip im Sozialversicherungsbeitrag, in: SDSRV Bd. 35 (1992), S. 65, 75; Rolfs, Das Versicherungsprinzip im Sozialversicherungsrecht, 2000, S. 462.

73 Im Hinblick auf Art. 74 Abs. 1 Nr. 12 GG einerseits und Art. 2 Abs. 1 GG andererseits.

74 So Papier/Möller, Die Rolle des Solidarausgleichs in der gesetzlichen Unfallversicherung, NZS 1998, S. 353, 359.

75 Vgl. Papier/Möller, Verfassungsrechtliche Fragen der Festsetzung der Beiträge in der Unfallversicherung, SGb 1998, S. 337 ff.

76 Art. 49 EGV; parallele Fragen stellen sich dann hinsichtlich der Anwendung der gemeinschaftsrechtlichen Wettbewerbsvorschriften, Art. 81 ff. EGV.

77 So grundl. Giesen, Sozialversicherungsmonopol und EGV, 1995.

78 Vgl. oben, I.

79 Auf diesen Aspekt wird noch zurückzukommen sein, vgl. unten III. 3. b) aa).

80 So auch Sächs. LSG, Breith. 2002, S. 791 ff.

81 Allerdings ist das nicht zwingend; vielmehr können grundsätzlich auch die Altlasten in die Berechnung einbezogen werden, vgl. Ricke, in: KassKomm., § 157 SGB VII, Rdnr. 16.

82 Vgl. eingehend Schulz, SGb 1999 (Fußn. 65), S. 172, 173, 176. 
träge der gewerblichen Berufsgenossenschaften ist es deshalb durchaus zutreffend, die Berufsgenossenschaften als „große Gefahrengemeinschaften“ zu bezeichnen. ${ }^{83}$

\section{Verfassungsrechtliche Vorgaben für die Bildung von Gefahrtarifen}

Allerdings gibt es im Einzelnen immer wieder Streit darüber, welche Unternehmen den einzelnen Berufsgenossenschaften zuzuordnen sind, und auch, wie innerhalb einer Berufsgenossenschaft Solidarität und Risikobezug zu verteilen sind. Der in den letzten Jahren wichtigste Beispielsfall ist die Einstufung der gewerblichen Arbeitnehmerüberlassung.

Konkret geht es um die Frage, ob es reicht, für diesen großen und amorphen Bereich gewerblicher Tätigkeiten nur zwei, zwangsläufig sehr grob geschnittene Tarifstellen zu bilden, oder ob stärker nach den konkreten Tätigkeiten der überlassenen Arbeitnehmer differenziert werden muß. Die gesetzlichen Vorgaben sind insofern offen. Sie regeln nicht, nach welchen Kriterien Gefahrgemeinschaften zu bestimmen sind. Insofern besteht für die Träger ein Spielraum, der als solcher mit der Verfassung, insbesondere dem Bestimmtheitsgebot und dem Parlamentsvorbehalt zu vereinbaren ist.

Mit der angesprochenen Problematik mußte sich, wie zu erwarten war, unlängst das Bundesverfassungsgericht auseinandersetzen. ${ }^{84}$ Es hat zwar die erhobene Verfassungsbeschwerde nicht zur Entscheidung angenommen, ${ }^{85}$ aber doch zugleich einige Hinweise zur Bildung von Gefahrtarifen gegeben. Bemerkenswerterweise hat die entscheidende Kammer des Gerichts dabei auch darauf abgestellt, daß sich das System in der Praxis bewährt habe und bis auf wenige Ausnahmen keine Härtefälle bekannt worden sind ${ }^{86}$ was auf den ersten Blick als eher pragmatisch denn dogmatisch abgesichertes Argument erscheint, hinter dem sich aber die Frage nach der Zulässigkeit von Typisierungen verbirgt. Denn im Mittelpunkt des Streits stand und steht die Reichweite des Art. 3 Abs. 1 GG und in diesem Zusammenhang vor allem die Notwendigkeit zu differenzierenden Regelungen.

Weiterführend sind denn auch die relativ knappen Aussagen zum Gleichbehandlungsgebot. Das BVerfG hat insofern dem Argument, es bedürfe einer möglichst genauen Abbildung der Risiken innerhalb der Gefahrtarife, eine Absage erteilt. Schon weil es

83 Schulz, a.a.O., S. 173, wonach die auf die unterschiedlichen Gefährdungsrisiken zwischen den jeweils erfassten Unternehmen zurückzuführenden Durchschnittsbeiträge bei den BG 1997 von 0,63 $\%$ bis $16,61 \%$ reichten.

84 BVerfG [Kammer] v. 3.7.2007, 1 BvR 1696/03 (www.bverfg.de) $=$ NZS 2008, S. $144=$ SozR 4$2700 \S 157$ Nr. $3=$ DVB1. 2008, S. 1172. Vgl. auch zum vorangegangenen Beschluß über die Besorgnis der Befangenheit eines Richters BVerfG [Kammer] v. 25.5.2007, 1 BvR 1696/03.

85 Gem. § 93a Abs. 2 BVerfGG mit der Begründung, die aufgeworfenen verfassungsrechtlichen Fragen könnten anhand der vorliegenden Rechtsprechung des Bundesverfassungsgerichts beantwortet werden.

86 BVerfG, a.a.O., Rdnr. 30. 
verläßlicher Statistiken bedarf, um der Tarifgestaltung eine ausreichend abgesicherte faktische Grundlage zu verschaffen, dürfen die Träger der Unfallversicherung nach Ansicht des BVerfG von einem relativ groben Raster ausgehen. Die in den letzten Jahren feststellbare Tendenz, keine reinen Tätigkeitstarife mehr zu bilden, weil dies den Entwicklungen auf dem Arbeitsmarkt nicht mehr entspräche, ist damit akzeptiert worden. 87 Mit anderen Worten: Zugunsten einer gesicherten kalkulatorischen Basis und zulasten des Risikobezugs darf bei der Festlegung der Beitragslast der finanzielle Ausgleich betont werden. Das Solidaritätsprinzip ist in diesem Sinne nicht gegenüber dem der Individualäquivalenz nachrangig.

\section{Zur Einführung von Kapitaldeckungselementen und deren Bewertung}

\section{Ansätze für eine Kapitaldeckung}

Wie könnte eine Kapitaldeckung in der Unfallversicherung erfolgen? Denkbar ist es, im Wege der Bildung von Rücklagen die finanziellen Mittel auf die Seite zu legen, die zur Deckung dauernder Lasten benötigt werden. Genauer gesagt geht es also um die Deckung von Anwartschaften ${ }^{8}$ auf Renten. Das kann auf unterschiedliche Weise geschehen: Indem etwa zunächst nur kleinere, einer Abfindung zugängliche Renten einbezogen werden, aber auch durch eine radikale Umstellung des gesamten Systems.

Was genau soll mit der Kapitaldeckung erreicht werden? Es geht um die bereits genannten möglichen Vorteile dieses Finanzierungsverfahrens, ${ }^{89}$ wobei ein gesamtwirtschaftliches und ein verteilungspolitisches Argument auseinander zu halten sind. Bezogen auf den erstgenannten Punkt, stellt die Einführung der Kapitaldeckung ein Mittel dar, um ganz allgemein die Zukunftsfestigkeit der Finanzierung angesichts der demographischen Veränderungen sicherzustellen. Sie hilft, die zu schulternden Lasten zu beziffern und die notwendigen Mittel beiseitezulegen und durch Zinserträge die künftigen Lasten zu decken. Abgesehen davon, daß diese Deckung aber ebenfalls von der Produktivität der künftigen Gesellschaft abhängt, entzieht sie allerdings zunächst den Unternehmen das für die Umstellung des Finanzierungsverfahrens erforderliche Kapital. 90

Zum anderen kann die Kapitaldeckung sehr viel konkreter helfen, ein Problem zu verringern, daß durch die Gliederung der gesetzlichen Unfallversicherung in verschiedene Träger nach einzelnen Gewerbezweigen hervorgerufen werden kann. Bei struktu-

87 BVerfG, a.a.O., Rdnr. 37 f.

88 Vgl. zu den Finanzierungsverfahren nur Schmähl, Ökonomische Grundlagen sozialer Sicherung, in: v. Maydell/Ruland (Hrsg.), SRH, 3. Aufl. 2003, S. $140 \mathrm{ff}$.

89 Vgl. oben, II. 1.

90 Diese Erkenntnis ist nicht neu; vgl. bereits das Heubeck-Gutachten (Fußn. 24), S. 51 ff. 
rellen Veränderungen hat eine schrumpfende Branche mit steigenden, weil in der Vergangenheit begründeten Leistungsausgaben zu kämpfen. Die einmal begründeten Rentenlasten sind grundsätzlich innerhalb eines Gewerbezweigs über Jahrzehnte zu decken. Verringert sich die Zahl der Unternehmen in einem solchen Zweig, wächst deren Belastung unverhältnismäßig. In Deutschland war davon zunächst der Bergbau betroffen. Die Situation führte dazu, daß 1968 erstmals ein Ausgleichsverfahren zwischen den gewerblichen Berufsgenossenschaften eingeführt wurde - durch das Gesetz zur Verwirklichung der mehrjährigen Finanzplanung des Bundes (FinÄndG) ${ }^{91}$ vom 21.12.196792, das mit Wirkung ab dem 1.1.1968 das Unfallversicherungs-Neuregelungsgesetz (UVNG) vom 30.4.196393 änderte (Art. $3 \S \S 1-6$ UVNG). ${ }^{94}$ Zuvor hatte $\S 723$ II RVO eine Ausgleichspflicht des Bundes für die Neulasten der Bergbau-BG vorgesehen. ${ }^{95}$ Danach bestand mit Art. 3 UVNG ${ }^{96}$ zugleich eine gemeinsame Pflicht der gewerblichen Berufsgenossenschaften zur Tragung der Bergbau-Altlast. 97 Die Regelungen wurden mehrfach geändert und gerieten vor einigen Jahren wieder in eine stärkere Diskussion. ${ }^{98}$ Anlaß waren die wirtschaftlichen Schwierigkeiten der Baubranche, Ergebnis war eine relativ moderate Reform des Lastenausgleichs. ${ }^{99}$ Angesichts des Umstandes, daß ähnliche Schwierigkeiten auch in Zukunft wieder auftreten können, erscheint die Kapitaldeckung als ein im Sinne des Wortes radikalerer Ausweg: Sie könnte den Lastenausgleich zwischen den gewerblichen Berufsgenossenschaften mehr oder

91 Art. 2 § 4 FinÄndG.

92 BGBl. I, S. 1259.

93 BGBl. I, S. 241.

94 Zur Mitwirkung des HVBG an der gesetzlichen Neuregelung Lauterbach, Finanzausgleich in der fachlich gegliederten Unfallversicherung, in: FS für Jantz, 1968, S. 107, 111.

95 In der zwischen 1965 und 1967 geltenden Fassung; auf dieser Grundlage wurden Zuschüsse gewährt, die als Anerkennung einer „Garantiehaftung des Bundes“ angesehen werden, so jedenfalls Kranz, Die Bundeszuschüsse zur Sozialversicherung, 1998, S. 72. Vgl. dazu Schulz, in: Wannagat (Hrsg.), SGB VII, Stand 2002, § 176, Rdnr. 7, wonach dieses Verfahren deshalb nicht befriedigte, weil der Bund Subventionen leisten musste, das Verfahren auf den Bergbau zugeschnitten war und zudem wegen der weiteren Schrumpfung dieses Wirtschaftszweigs die zu zahlenden Ausgleichsbeträge nicht ausreichten. Nachweise dazu bei Schimanski, Risiko- und Solidaritätsausgleich in der gesetzlichen Unfallversicherung, Diss. Bochum 1986, S. 68 ff.

96 Die Norm war bei ihrer Einführung sehr umstritten; die Begrenzung des Ausgleichs auf die Rentenlast ging auf Einwände des HVBG zurück, vgl. Gotzen/Doetsch, Kommentar zur Unfallversicherung, 1963, Anm. zu Art. $3 \S \S 1-3$ UVNG. Umfang des Ausgleichs: 234 Mio. DM jährlich, was nach Vorberechnungen des HVBG zu einer Beitragserhöhung um etwa 0,22 DM auf je $100 \mathrm{DM}$ Lohnsumme führen sollte, vgl. Gotzen/Doetsch, Kommentar zur Unfallversicherung, 1963, Anm. zu $\S § 737-739$.

97 Vgl. zur Entstehung der Ausgleichsregelungen näher Schimanski, Risiko- und Solidaritätsausgleich in der gesetzlichen Unfallversicherung, in: FS für Waltermann, 1996, S. 127, 128 ff.

98 Vgl. dazu Becker, Der Finanzausgleich in der gesetzlichen Unfallversicherung, Zur Lastenverteilung zwischen den gewerblichen Berufsgenossenschaften und deren Reform, 2004.

$99 \S 176$ ff. idF. durch das G zur Änderung des SGB und anderer Gesetze v. 24.7 .2003 (BGBl. I, S. 1526). Dazu Wollschläger/Kossens, Das Gesetz zur Änderung des Sozialgesetzbuches vom 27.6.2003 und anderer Gesetze, NZS 2003, S. 514 ff. 
weniger entbehrlich werden lassen. ${ }^{100}$ Allerdings schließen sich natürlich beide Lösungswege keineswegs gegenseitig aus, sondern sie können, wie im Entwurf des UVMG vorgesehen, ${ }^{101}$ gemeinsam beschritten werden.

\section{Normative Vorgaben}

\section{a) Verteilung in der Zeit: Generationengerechtigkeit}

Zunächst hat die Einführung eines Kapitaldeckungsverfahrens viel mit der Gerechtigkeit in einer zeitlichen Dimension zu tun. Denn dieses Verfahren ermöglicht die Zurechnung von Lasten zu den Verursachern, zumindest grundsätzlich, wobei die Geldentwertung allerdings bei der genauen Zumessung Schwierigkeiten bereitet. Das ist ein Argument, das insbesondere in der Renten-, aber nicht weniger auch in der Krankenund Pflegeversicherung eine große Rolle spielt. ${ }^{102}$

Rechtlich gesehen allerdings läßt sich eine Gleichheit in der Zeit nur sehr eingeschränkt fordern. ${ }^{103}$ Soll der Gesetzgeber nicht auf Dauer blockiert werden, muß der Grundsatz gelten, daß er nur für seine konkreten Maßnahmen Gleichbehandlung schuldet, Änderungen sich also nicht an der Behandlung in der Vergangenheit messen lassen. Allerdings ist damit noch nichts gesagt über die Zulässigkeit der Verschiebung von Lasten in die Zukunft. Eine solche Verschiebung ist notwendigerweise schon mit der Einführung eines umlagefinanzierten Sicherungssystems verbunden. Der Vorteil dieser Systeme ist bekanntlich der, daß ohne den zeitaufwendigen Aufbau eines Kapitalstocks dem Sicherungsanliegen genügt und deshalb unmittelbar auf gegenwärtige soziale Bedarfslagen reagiert werden kann. Der Nachteil ist der, daß kommende Generationen zur Zahlung der einmal angelegten finanziellen Lasten verpflichtet werden. Der damit verbundene finanzielle Ausgleich zwischen Angehörigen verschiedener Generationen spielt insbesondere bei Rentenleistungen eine Rolle. ${ }^{104}$ Und das Umlageverfahren berührt zugleich das Verhältnis zwischen den kommenden Generationen, weil deren Belastung nicht nur von der Höhe der gezahlten Leistungen, sondern ganz wesentlich von

100 In diesem Sinn Breuer, in: verdi (Hrsg.), Die Diskussion zur Reform der gesetzlichen Unfallversicherung, 2007, S. 20, 28.

101 Vgl. näher oben, II. 2.

102 In den letztgenannten Zweigen allerdings im Hinblick auf die jeweils unterschiedliche zeitliche Verbindung zwischen Beitragszahlung und Leistungsbezug nicht im gleichen Maße; soweit aber davon auszugehen ist, daß Leistungsansprüche mit dem Alter typischerweise steigen, führt eine Alterung der Gesellschaft dazu, daß anstelle des intrapersonalen (über den jeweiligen Lebenslauf zu berechnenden) der intergenerationelle Ausgleich an Bedeutung gewinnt.

103 Vgl. dazu nur Becker, Generationengerechtigkeit als juristisches Problem, in: Generationengerechtigkeit - Inhalt, Bedeutung und Konsequenzen für die Alterssicherung, DRV-Schriften Bd. 51 (2004), S. 56 ff. m.w.N.

104 Vgl. aber auch Fußn. 102. 
dem im Leistungszeitraum bestehenden Verhältnis zwischen Beitragszahlern und Leistungsempfängern abhängig ist.

Obwohl also schon die Einführung eines umlagefinanzierten Sicherungssystems mit Belastungen verbunden ist und deshalb der Gleichheitssatz als Maßstab für die Überprüfung entsprechender Errichtungsgesetze dienen kann, wird bis jetzt höchstens die Ausgestaltung entsprechender Systeme unter dem Gesichtspunkt der Generationengerechtigkeit diskutiert. ${ }^{105}$ Das mag dem Umstand geschuldet sein, daß die entsprechenden Fragen erst angesichts der aktuellen demographischen Entwicklungen an Gewicht gewinnen. Es ist aber auch in der Sache verständlich. Denn zum einen stellt ein umlagefinanziertes Sicherungssystem jedenfalls im Grundsatz ein probates Mittel dar, um den aus dem Sozialstaatsprinzip (Art. 20 Abs. 1, 28 Abs. 1 GG) ableitbaren Gesetzgebungsaufträgen nachzukommen; insbesondere besteht bei der Wahl des Finanzierungsverfahrens schon angesichts der mit jedem Verfahren verbundenen Chancen und Risiken ein Einschätzungsspielraum des Gesetzgebers. Zum anderen sind aber auch die genannten ökonomischen Zusammenhänge juristisch stark relativiert worden. Denn die Sozialversicherungsbeiträge sind Vorzugslasten. Gilt auch für deren Kalkulation schon wegen des sozialen Ausgleichs nicht eine strenge Individualäquivalenz, ${ }^{106}$ so doch das Erfordernis, daß der Beitragszahlung Leistungen gegenüberstehen müssen. ${ }^{107}$ Bei Ansparleistungen wird zudem der Leistungsanspruch eigentumsrechtlich geschützt, 108 so daß die Beitragszahler auch in umlagefinanzierten Systemen eine eigene, rechtlich gesicherte Position erhalten. Deshalb stellen sich Fragen der Gleichbehandlung weniger, weil nachfolgende Generationen durch ihre Beiträge die Rentenleistungen der ersten Generation zahlen müssen, sondern vielmehr im Hinblick auf das wahrscheinlich je nach Generation unterschiedliche und künftig für die kommenden Generationen sich verschlechternde Verhältnis zwischen der Höhe der Beitragszahlung und der Höhe des Leistungsanspruchs. $\mathrm{Da} \beta$ aber auch insofern keine einfachen Rechnungen möglich und Verschlechterungen nicht per se unzulässig sind, sei an dieser Stelle nur erwähnt. 109

105 Vgl. neben dem in Fußn. 103 genannten Sammelband etwa Steiner, Generationenfolge und Grundgesetz, NZS 2004, S. 505 ff.

106 Vgl. dazu oben, III. 2.

107 Vgl. etwa zu Einmalzahlungen BVerfGE 102, 127; zur anderen Lage bei Förderungsleistungen (Arbeitslosenhilfe) BVerfG [Kammer], NZS 2006, S. 247.

108 Grundl. BVerfGE 53, 257, 290 ff.; zur Rentenversicherung näher Becker, Verfassungsrechtlicher Schutz rentenrechtlicher Positionen, LVA Mitt. 2005, S. 228 ff.; Ebsen, Das Verfassungsrecht als Steuerungsinstrument für die Balance zwischen Bestandssicherung und Flexibilität in der gesetzlichen Rentenversicherung, in: Becker u.a. (Hrsg.), Alterssicherung in Deutschland, 2007, S. 81, 86 ff.

109 Näher dazu Becker, DRV-Schriften Bd. 51 (2004) (Fußn. 103). 


\section{b) Horizontale Verteilung: Abgrenzung der Solidargemeinschaft}

Zunächst besagt ein Übergang zu einer Kapitaldeckung nichts über die horizontale Verteilung der aufzubringenden Lasten. Grundsätzlich könnten die Rückstellungen in einen gemeinsamen Fonds aller Träger eingestellt werden. Sie könnten aber auch in den jeweiligen Haushalten der einzelnen Träger vorgenommen werden. Die letztgenannte Variante ist diejenige, die der Konstruktion der Haftungsablösung durch die Unfallversicherung am besten entspricht. Und sie ordnet im Sinne der Äquivalenz die Lasten den Verursachern auf. Sie entspräche also dem bestehenden System der Gliederung nach Branchen und der Einheit von Prävention, Rehabilitation und Kompensation.

Allerdings genießt selbst im bestehenden System die Kapitaldeckung rechtlich gesehen nicht zwingend einen Vorrang gegenüber dem Finanzausgleich. Zwar kann unter dem Gesichtspunkt der Systembindung 110 eine Verpflichtung des Gesetzgebers abgeleitet werden, möglichst wenig von einem bestehenden System abzuweichen. ${ }^{111}$ Für die Beachtung der Systemkonsistenz spricht in diesem Sinne ein allgemeines Prinzip, für Abweichungen gilt umgekehrt ein Begründungszwang und darüber hinaus auch die Forderung, nur das mildeste Mittel i.S. der kleinstmöglichen Abweichung einzusetzen. Aber zum einen kann die Einhaltung dieses Grundsatzes nur relativ grob überprüft werden, die Prüfungsintensität müßte wegen der für die Durchführung von Reformen notwendigen Gestaltungsfreiheit gering bleiben. Zum anderen spricht die Systemkonsistenz innerhalb der bestehenden Unfallversicherung nur grundsätzlich für eine branchenbezogene Solidarität. Wie diese am besten zu erreichen ist, bleibt offen. Da eine Umstellung des Finanzierungsverfahrens im Ergebnis nicht nur mit Unsicherheiten, sondern auch mit neuen Belastungen verbunden ist, kann sie nicht als ein evident milderes Mittel angesehen werden. ${ }^{112}$ Zudem kann zwar mit der Kapitaldeckung - wie beim Finanzausgleich - die Zusammenlegung von Trägern vermieden werden. Aber auch insofern sind zwei Einwände zu bedenken. Erstens wird sich der Finanzausgleich zwischen den Trägern nicht völlig vermeiden lassen. Für eine Übergangszeit ist das ohne Weiteres einsichtig. Aber auch bei völliger Umstellung auf eine Kapitaldeckung dürfte es nicht ausgeschlossen sein, daß die Belastung einzelner Träger unverhältnismäßig hoch

110 Dazu grundl. Degenhart, Systemgerechtigkeit und Selbstbindung des Gesetzgebers als Verfassungspostulat, 1976; vgl. auch Peine, Systemgerechtigkeit, 1985.

111 Zum Sozialrecht: Becker, Selbstbindung des Gesetzgebers im Sozialrecht - Zur Bedeutung von Konsistenz bei der Ausgestaltung von Sozialversicherungssystemen, in: FS 50 Jahre BSG, 2004, S. 77 ff.; Axer, Kontinuität durch Konsequenz in der Sozialversicherung, in: FS für Isensee, 2007, S. $965 \mathrm{ff}$.

112 Müssten hingegen die systemkonformen Spielräume wegen der wirtschaftlichen Entwicklung überschritten werden, hat der Gesetzgeber die Wahl, entweder das gegliederte System grundsätzlich zu ändern oder, sofern er dies für ausreichend halten würde, nur das Finanzierungsverfahren umzustellen. Anderes würde natürlich gelten, wenn man annehmen wollte, da $\beta$ - entgegen der bisherigen Rspr. des BVerfG - ein vollständiger sozialer Ausgleich zwischen allen gewerblichen Unternehmern unzulässig wäre. 
werden kann. Zweitens finden Fusionen bereits statt und werden weiterhin angestrebt, ${ }^{113}$ so daß die Grenzen des zu beachtenden Systems nicht mehr klar zu ziehen sind.

\section{Zu der eingeleiteten Neuregelung}

Wenn auch der Gesetzgeber nach alledem nicht verpflichtet ist, kapitalgedeckte Elemente in die gesetzliche Unfallversicherung einzuführen, so sprechen doch für eine solche Entscheidung zwei rechtliche Prinzipien, nämlich die Generationengerechtigkeit und die Systemkonsistenz. Angesichts der Tatsache, daß mit Umbauten auf Finanzierungsseite immer zugleich Lastenverschiebungen verbunden sind, ist es geboten, Übergangsregelungen vorzusehen und den Umbau nur schrittweise zu vollziehen. Die entsprechenden Schritte sollten bald unternommen werden. Dabei muß insbesondere die sozialpolitisch desaströse, wenn auch politökonomisch verständliche Haltung verabschiedet werden, in guten Zeiten Leistungen zu versprechen, die dann in schlechten Zeiten zu kürzen sind. Zusätzliche, mit jedem Umbau verbundene Belastungen lassen sich besser (er)tragen, wenn die wirtschaftliche Lage dafür Raum läßt.

Insofern enthält der Entwurf eines Gesetzes zur Modernisierung der gesetzlichen Unfallversicherung (UVMG) die richtigen Schritte. Er verbindet eine die Systementscheidungen grundsätzlich bewahrende Lastenverteilung mit dem zukunftsweisenden Aufbau einer teilweisen Kapitaldeckung für Rentenlasten. Die Einzelheiten bedürfen noch der Festlegung. Über sie läßt sich auch weiterhin trefflich streiten. ${ }^{114}$ Denn die genannten rechtlichen Prinzipien geben zwar eine Richtung vor, sind aber dennoch offen genug, um dem Gesetzgeber ausreichenden sozialpolitischen Gestaltungsspielraum zu lassen. Er steht damit zugleich in der politischen Verantwortung, bei deren Wahrnehmung er sich von den Selbstverwaltungsträgern beraten lassen kann, die er aber nicht völlig an diese abgeben darf.

113 Vgl. zum UVMG oben, II. 2. Vgl. in diesem Zusammenhang auch $§ 118$ Abs. 4 SGB VII des Entwurfs.

114 Vgl. etwa zu den Kriterien für die Bestimmung des jeweiligen Anteils an der gemeinsamen Lastentragung die Stellungnahme des BDS, http://www.bds-dgv.de/wDeutsch/pressezentrum/aktuell/pe20080213.shtml. 


\title{
Vor- und Nachteile der verschiedenen Finanzierungsformen der Arbeiterunfallversicherung: Beobachtungen auf ausgesuchten Märkten
}

\author{
Dr. med. Héctor Upegui
}

\begin{abstract}
Bevor man in eine Diskussion über die verschiedenen Finanzierungsarten der Arbeiterunfallversicherung einsteigen kann, sollte man sich die Organisationsformen dieser Versicherung vor Augen führen. Zunächst werde ich erörtern, welche Formen der Gruppenbildung grundsätzlich in Frage kommen und warum diese bei der Arbeiterunfallversicherung wenig sinnvoll sind. Danach werde ich einige Modelle für die Abgrenzung der einzelnen Gruppen vorstellen, die wir in unserer Forschungsgruppe entwickelt haben. Schließlich behandele ich die so genannte „Trennung“ von Risiko und Finanzierung sowie die möglichen Konsequenzen und Herausforderungen, die für die verschiedenen Systeme der Arbeiterunfallversicherung damit verbunden sind.
\end{abstract}

\section{Modelle für die Arbeiterunfallversicherung}

Die große Vielfalt an Organisationsformen der Arbeiterunfallversicherung in den verschiedenen Teilen der Welt erweist sich als Schwierigkeit, wenn man homogene Gruppen bilden will, von denen man Ähnlichkeiten oder Unterschiede ableiten möchte. Darauf ist auch zurückzuführen, dass die Diskussion sich in der Vergangenheit und noch in der Gegenwart häufig auf die Besonderheiten in einzelnen Ländern oder auf die Herangehensweisen beschränkt, die in anderen Sparten der Sozialversicherung, wie beispielsweise in der Rentenversicherung, als Standard gelten, die aber für die Arbeiterunfallversicherung nicht sinnvoll sind.

Um also ein oder mehrere Modelle für die Arbeiterunfallversicherung identifizieren zu können, ist es einfacher mit den Kriterien anzufangen, die sich bei diesem Versicherungstyp nicht für die Gruppenbildung eignen:

- öffentlich-rechtliche im Gegensatz zu privatrechtlicher Ausgestaltung

- Umlageverfahren (pay-as-you-go) im Gegensatz zum Kapitaldeckungsverfahren (fully funded)

- Vorliegen beziehungsweise Fehlen von Gewinnerzielungsabsicht

- liberalisiertes im Gegensatz zu einem regulierten Marktumfeld

Diese Kriterien für die Gruppenbildung sind für die Arbeiterunfallversicherung ungeeignet und erlauben es nicht, adäquate Vergleiche zu ziehen. Es ist nichts Neues, dass 